\title{
STUDY OF THE PHASE STRUCTURE OF AN SU(3) HIGGS MODEL AT FINITE TEMPERATURE
}

\author{
F. $\mathrm{KARSCH}^{1}$ \\ CERN, CH 1211 Geneva 23, Switzerland
}

\author{
E. SEILER ${ }^{2}$ \\ Max Planck Institut für Physik und Astrophysik - Werner Heisenberg Institut für Physik - \\ D 8000 Munich 40, Fed. Rep. Germany
}

and

\section{I.O. STAMATESCU}

Institut für Theorie der Elementarteilchen, Freie Universität Berlin, D 1000 Berlin, Germany

Received 18 February 1985

\begin{abstract}
We analyse numerically an SU(3) Higgs model with complete symmetry breaking and radial degree of freedom on asymmetric, periodic lattices. The character of both the Higgs and deconfining transitions is found to depend on the Higgs self-coupling and on a parameter which may simulate the number of flavours. In particular, an increase in the latter leads to the disappearance of the deconfining transition for small Higgs masses.
\end{abstract}

1. Introduction. The phase structure of gauge models with Higgs fields that "break the symmetry completely" in the traditional perturbative language still remains insufficiently understood. The main reason is the so-called duality [ $1-3]$ between confinement and the Higgs mechanism and - closely related to it - the nonexistence of a simple order parameter characterizing the Higgs ("broken symmetry") phase unambiguously.

On the other hand the popular "inflationary scenarios" for the very early universe [4,5] rely in an essential way on the existence of a first-order transition between the "broken" and "unbroken" phases at

t Presented at the "Lattice co-ordination meeting", CERN, Geneva, December 1984.

1 Present address: University of Illinois, Urbana, IL 61801, USA.

2 Present address: Princeton University, Princeton, NJ 08544, USA. least at high temperature. Earlier Monte Carlo searches for such a phenomenon in SU(2) models both with adjoint $[6,7]$ and fundamental Higgs $[8,9]$ do not always give unambiguous evidence of its occurrence. In this paper we go from SU(2) to SU(3).

For this group it is not quite as straightforward to "completely break the symme try" as for SU(2); a single fundamental SU(3) Higgs field would still leave an $S U(2)$ subgroup unbroken. What would be needed is a "flavour" multiplet of fundamental Higgs fields. On the other hand, in the case of SU(2), "complete breakdown" is achieved by a single fundamental Higgs field which can also be described by an SU(2) matrix, together with a radial degree of freedom if desired. This suggests to try to achieve "complete breakdown" in SU(3) also by choosing for the Higgs fields multiples of SU(3) matrices. At first we thought that this choice would not be in any essential way different from a conventional model with a 
multiplet of Higgs fields. The often surprising results we are describing in this article, however, make this belief rather doubtful. In particular the model shows a wealth of first-order transitions, some of the sort that that should be liked by inflationary cosmologists. We are not sure, however, to what extent these phenomena are artifacts of the model. We are currently checking this by running a similar study of a model with a more conventional Higgs multiplet [10].

That our model might show some peculiar features could perhaps be expected because, unlike in the case of $\mathbf{S U}(2)$, the multiples of SU(3) matrices do not form a linear space, so a perturbative analysis is not easily possible. The smallest linear space in which our Higgs fields could be imbedded is the space of all complex $3 \times 3$ matrices [11]; the restriction to the multiples of SU(3) matrices can be thought of as arising as a limiting case of a rather complicated potential.

2. The model. Our model has the following degrees of freedom: The usual gauge fields (link variables $\left.\left\{U_{\mu}(x)\right\}, U_{\mu}(x) \in \mathrm{SU}(3)\right)$; Higgs fields living on sites, consisting of radial and internal degrees of freedom $\{R(x), V(x)\}(V(x) \in \mathrm{SU}(3))$.

The action we choose has the form

$$
\begin{aligned}
S= & -\frac{2 \kappa}{3} \sum_{x, \mu} \operatorname{Re} \operatorname{Tr} R_{x} V_{x}^{+} U_{x, \mu} V_{x+\mu} R_{x+\mu} \\
& +\sum_{x} R_{x}^{2}+\lambda \sum_{x}\left(R_{x}^{2}-1\right)^{2}-\frac{\beta}{3} \sum_{\text {Plaq. }} \operatorname{Re} \operatorname{Tr} U_{\partial \mathrm{P}} .
\end{aligned}
$$

This action contains the parameters $\kappa, \lambda, \beta$ corresponding to the Higgs mass, coupling constant of the Higgs self-interaction and gauge coupling constant.

In order to fully specify the model we have to give a probability measure on the field configurations. This will be given by

$$
Z^{-1} \mathrm{e}^{-S} \prod_{x, \mu} \mathrm{d} U_{x, \mu} \prod_{x} \mathrm{~d} V_{x} \prod_{x} R_{x}^{f} \mathrm{~d} R_{x} .
$$

$\mathrm{d} U_{\mu}, \mathrm{d} V$ denote the Haar measure on $\mathrm{SU}(3), \mathrm{d} R$ the Lebesgue measure on $[0, \infty)$. It should be noted that there is an additional free parameter $f$ appearing in this measure. Unlike the case of SU(2), where $f=3$ would lead to the standard fundamental Higgs model, here there is no canonical choice of $f$. We will look at various cases $f=3, f=8, f=17$ and $f=50$, and we will see that the phase structure is rather sensitive to this parameter. $f=8$ is suggested by the dimension of SU(3) and analogy with $\mathrm{SU}(2), f=17$ by the dimension of the space of complex $3 \times 3$ matrices. $f=$ 50 was chosen to have some comparison with QCD with fermions (see below). $f=3$ is just a small value.

To understand, at least roughly the effect of varying $f$, consider that there were a natural choice for it, $f=f_{0}$ say, leading to an effective potential without logarithmic terms (e.g. $f_{0}=3$ for $\mathrm{SU}(2)$ ). Then using the measure eq. (2) with $f \neq f_{0}$ simulates a term

$-\left(f-f_{0}\right) \ln R_{x}$

in the effective potential, producing additional structure at $R_{x}=0$. Due to quantum effects the logarithmic singularity at zero will be smeared. For $f>f_{0}$ we thus get a peak at $R_{x}=0$, resembling a Higgs potential, even if the original action eq. (1) has positive mass squared $\left(\kappa<\frac{1}{8}(1-2 \lambda)\right)$. For $f<f_{0}$ on the other hand, a Higgs-type potential $\left(\kappa>\frac{1}{8}(1-2 \lambda)\right)$ will develop a second deep at the origin which can lead to a first-order phase transition.

Similar effects have been observed for $\mathrm{SU}(2)$ with $f=0,3$ [9].

\section{Results. As remarked before our model has a} tendency to show first-order transition. As in QCD with fermions [12-14] at finite temperature and low $k$ we find persistence of the first-order deconfinement transition of the pure $\mathrm{SU}(3)$ gauge model. But we also find first-order transitions by varying $\kappa$ at fixed $\beta$, corresponding to a transition between "broken" and "unbroken" phases. Generally these transitions become weaker, sometimes become second order (or even disappear altogether) as we increase $f$ and/or $\lambda$. The limiting case $\lambda=\infty$ (corresponding to $R=1$ ) seems to show an at most second-order transition. On the other hand it is remarkable that at low $f(f=$ 3 ) and at $\lambda=0$ even the "spin model" $\beta=\infty$ has a first-order transition. This transition is not quite like the usual magnetizing transition in spin models. The main effect seems to be a transition between a phase in which $R_{x}$ fluctuates around small values to a phase in which $R_{x}$ fluctuates around a value of the order of $R_{\mathrm{cl}}$. Of course the phase with small $R_{x}$ values is at the same time disordered because the link term in the action is small for small $R_{x}$, whereas in the other phase we find spontaneous magnetization. So at the 
transition both $\left\langle R_{x}\right\rangle$ and the magnetization $\mathscr{m}$ jump.

This way of modelling ferromagnets with firstorder transitions ("Thouless effect") may be of some interest.

For $\lambda=0$ our models become unstable for $\kappa>\frac{1}{8}$. But there is a clear signal of a phase transition at $\beta=$ $\infty, k=\kappa_{\mathrm{c}}<\frac{1}{8}$, (at $\left.f=17\right)$. This transition also occurs for $\beta<\infty$ and seems to become actually stronger there.

Even more remarkable is the following phenomenon: For $f=8, \lambda=0.1$ we have a clear second-order transition in the spin model $(\beta=\infty)$. This transition, however, seems to become first order as we turn on the gauge coupling $(\beta<\infty)$ and then disappear again for very strong gauge coupling (the transition is definitely gone by $\beta=0.5$ but we did not try to localize the end point).

We summarize our results in four figures. The calculations have been done on the Cray-1 computers of IPP Garching and of WRB Berlin. We used vectorized programs as have been described in ref. [15]. The simulations have been done on lattices of size $N_{\mathrm{t}} N_{\mathrm{s}}^{3}$ with $N_{\mathrm{t}}=2, N_{\mathrm{s}}=4,6,8$. We take typically some thousands of sweeps per point.

Fig. 1a shows a rough phase diagram for $f=8, \lambda=$ 0.1 . We use the values of $R=\left\langle R^{2}\right\rangle$ and of the Polyakov loop expectation $P=\left\langle\operatorname{Re} \operatorname{Tr} \Pi_{t} U_{(\boldsymbol{x}, t), 0}\right.$ to distinguish three regions:

$R, P$ large : the Higgs region,

$R$ small, $P$ large : the deconfined region,

\section{$R, P$ small : the confinement region .}

Although $R, P$ are not order parameters, the various transitions are accompanied by jumps or strong variation in these expectations. Figs. 1b-1d show typical behaviour of various observables across the transition lines.

Here and in the following:

$A=\frac{1}{3} \beta\left\langle\operatorname{Re} \operatorname{Tr}\left(U_{\partial \mathrm{P}}\right)\right\rangle$,

$H=\frac{2}{3} \kappa\left\langle\operatorname{Re} \operatorname{Tr} R_{x} V_{x}+U_{x, \mu} V_{x+\mu} R_{x+\mu}\right\rangle$,

$\epsilon_{\mathrm{G}}=A$ (time-like plaquette)

$-A$ (space-like plaquette),
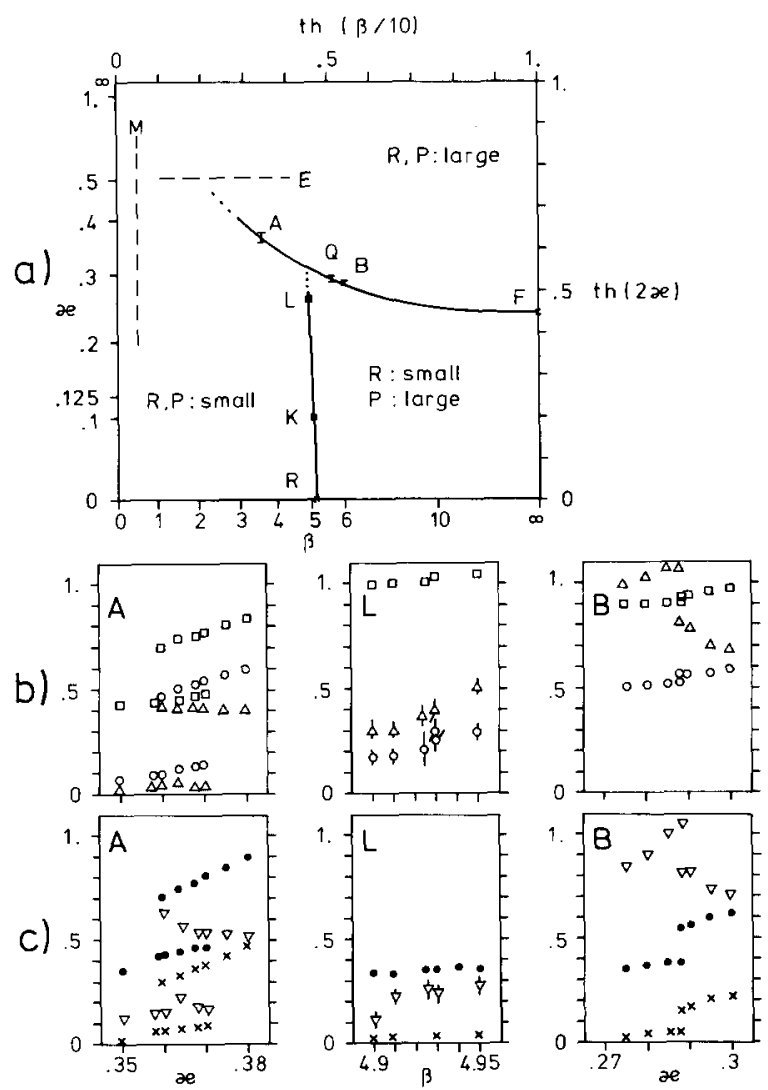

Fig. 1. General picture for $f=8, \lambda=0.1$. (a) Phase structure and transitions: Higgs and finite temperature. The capital letters $(A, B, E, \ldots)$ denote runs along $\beta=$ const. or $\kappa=$ const. lines, by which the transitions were localized: first-order transitions $(A, Q, B, R)$; second-order or weaker $(F, K, L)$; no transition (E, M). (b) The behaviour of some YM-observables across the transitions A, L, B: o: $P$; $\mathrm{\square}: A \times 1 / 2(\times 1 / 4$ for $\mathrm{B})$; $\Delta: \epsilon_{\mathrm{G}} \times 4$. (c) Same for some Higgs observables: $\bullet: R \times 1 / 10$; $\mathrm{X}: H \times 1 / 10 ; \nabla: \epsilon_{H} \times 4$.

$\epsilon_{\mathrm{H}}=H($ time-like link $)-H($ space-like link $)$,

$m=\left\langle\left|\frac{1}{N_{V}} \sum_{x} V_{x}\right|\right\rangle$,

where $N_{V}$ is the lattice volume.

Next we illustrate the fact of the Higgs transition as function of $f$, both for the spin model $(\beta=\infty$; fig. 2 ) and for the Higgs model $(\beta<\infty$; fig. 3). As noticed above, the transition of the spin model, which for $f=$ 8 is second order, becomes first order for $f=3$ while getting weaker for $f=17$. For $\beta<\infty$ the transition is 


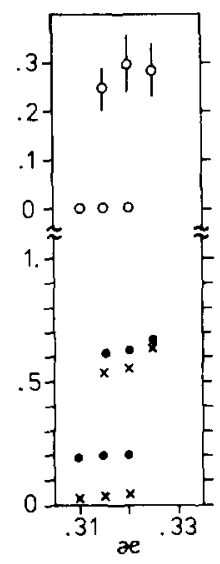

a)

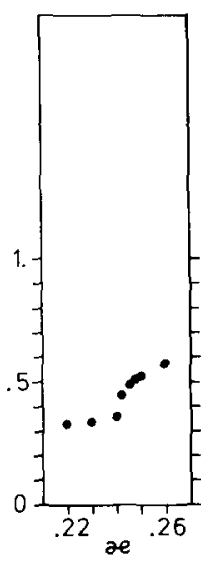

b)

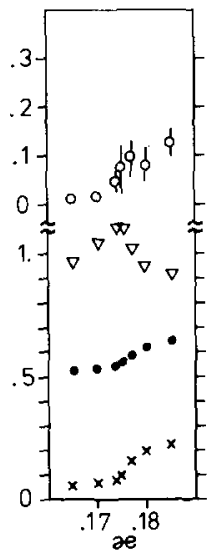

c)

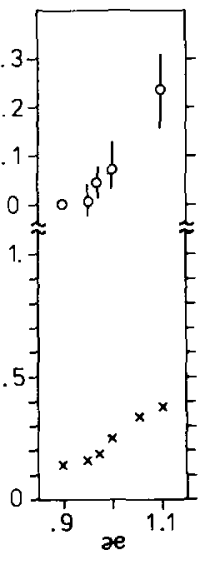

d)

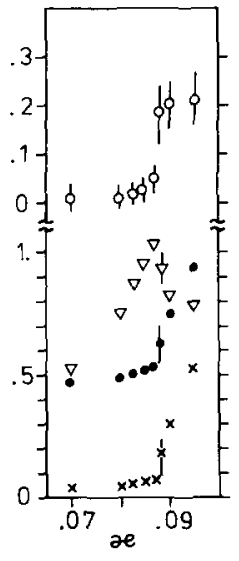

e)

Fig. 2. The spin model transition $(\beta=\infty)$. (a) $f=3, \lambda=0.1 ;$ (b) $f=8, \lambda=0.1 ;$ (c) $f=17, \lambda=0.1 ;$ (d) $R=1 ;$ (e) $f=17, \lambda=0$. Here: $\bullet: R \times 1 / 10(R \times 1 / 20$ for $(\mathrm{e})) ; \times: H \times 1 / 5 ; \nabla: \epsilon_{\mathrm{H}} \times 4 ; 0: \mathrm{m}$.

steeper becoming first order for $f=8$ while apparently remaining second order for $f=17$. Finally, going to $\lambda=0$ seems also to strengthen the transition, while fixing $R_{x}=1$ weakens it.

Finally we discuss the fate of the deconfining transition. For small $\kappa$ it is first order, weakening as expected with increasing $k$ (and also with increasing $f$

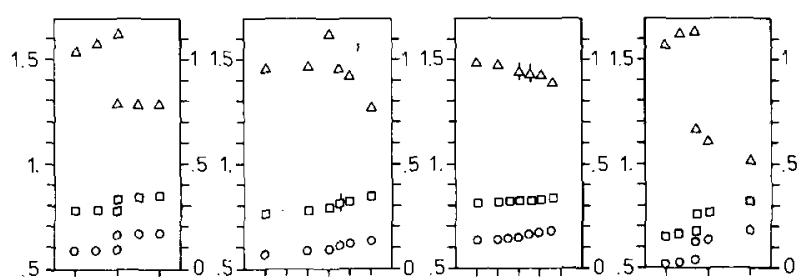

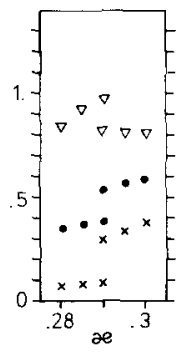

b)

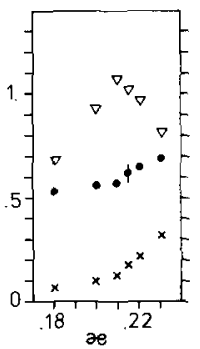

c)

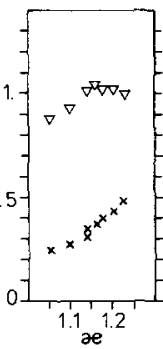

d)

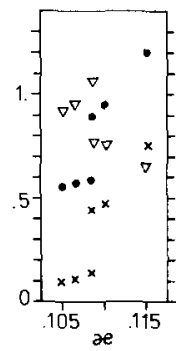

e)
Fig. 3. The Higgs transition at $\beta<\infty$. (b) $f=8, \lambda=0.1, \beta=5.6$ (Q); (c) $f=17, \lambda=0.1, \beta=5.6$; (d) $R=1, \beta=6$; (e) $f=17$, $\lambda=0, \beta=5.2$. Here $0: P ; \square: A=1 / 4 ; \Delta: \epsilon_{\mathrm{G}} \times 4$ (right scale); $\bullet: R \times 1 / 10(R \times 1 / 20$ for $(\mathrm{e})) ; \times: H \times 1 / 5 ; \nabla: \epsilon_{\mathrm{H}} \times 4$. and/or $\lambda$ ) see fig. 4 . In fig. 4 we present the situation for $f=50, \lambda=0$. The reason for using such a high value for $f$ is the following. To the lowest order in $k$ the effect of the Higgs fields can be obtained from an effective action with a Polyakov loop term (we consider lattices with $N_{\mathrm{t}}<4$ )

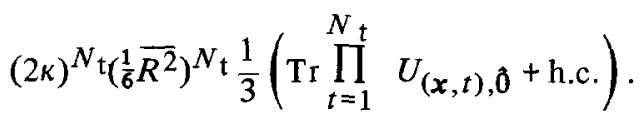

Here

$\overline{R^{2}}=\frac{\int \mathrm{d} r r^{f+2} \exp \left(-r^{2}\right)}{\int \mathrm{d} r r^{f} \exp \left(-r^{2}\right)}=\frac{f+1}{2}$.

To the same order in $\kappa$ QCD with $N_{\mathrm{f}}$ fermions produces a similar term [12]

$(2 \kappa)^{N_{\mathrm{t}} 2 N_{\mathrm{f}}}\left(\operatorname{Tr} \prod_{t=1}^{N_{\mathrm{t}}} U_{(\boldsymbol{x}, t), \hat{0}}+\right.$ h.c. $)$,

Thus to the lowest order in $\kappa$ the two models coincide if we take

$f=12\left(6 N_{\mathrm{f}}\right)^{1 / N_{\mathrm{t}}}-1$,

leading to $f \sim 50$ for $N_{\mathrm{f}}=3, N_{\mathrm{t}}=2$. Notice that we could not obtain the same effect by choosing a larger $\kappa$ because this latter is the expansion parameter. What we need is to introduce many more flavours and this we can simulate economically by choosing a large $f$.

The results in fig. 4 show the weakening of the 


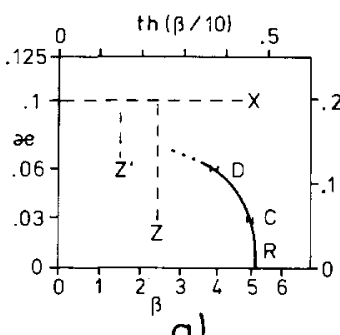

a)

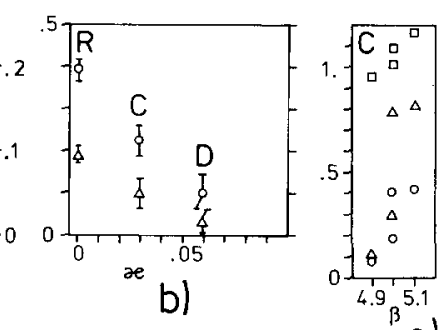

C)

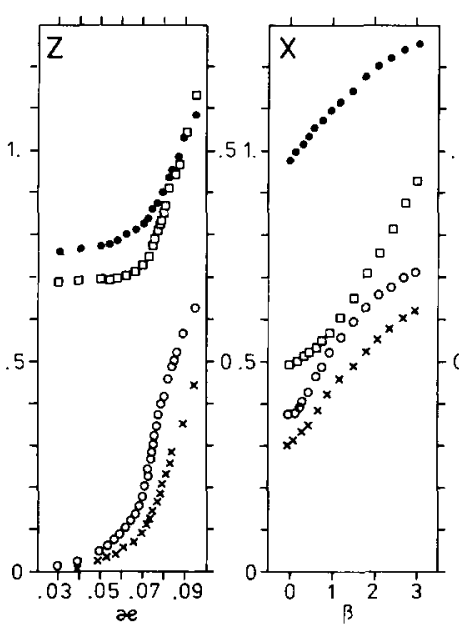

d)

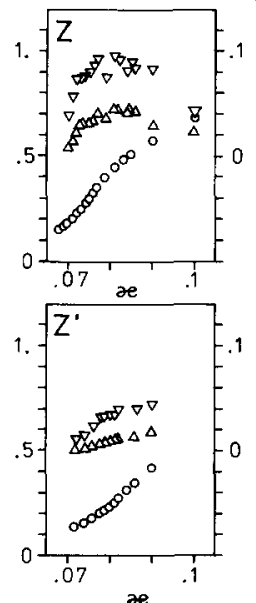

e)
Fig. 4. The deconfining transition for $f=50, \lambda=0$. (a) The transition line. (b) The jump in some observables as function of $\kappa$ (points $\mathrm{R}, \mathrm{C}, \mathrm{D}) ; 0: P ; \Delta: \epsilon_{\mathrm{G}}$. (c) The behaviour across the transition at $\mathrm{C}: \circ: P ; \square: A \times 1 / 2 ; \Delta: \epsilon_{\mathrm{G}} \times 4$. (d) The general behaviour along the lines $\mathrm{X}, Z . \circ: P ; \mathrm{X}: H \times 1 / 20$ (both left-hand scale); $\square: A \times 1 / 2, \bullet: R \times 1 / 50$ (both right-hand scale). (e) Cross-over region below $\beta=2.4 . \circ: P$; $\square: A ; \Delta: \epsilon_{\mathrm{G}}$; $\nabla: \epsilon_{\mathbf{H}}$

(for small $k$ still first order) deconfining transition up to its complete disappearance: the transition does not extend outside the region $\beta \geqslant 2.4, \kappa \leqslant 0.1$ (we did not try to localize precisely the end point). How- ever a kind of cross-over region may be observed for $\beta \leqslant 2.4$. The effect of bosonic matter on the deconfining transition for small masses confirms thus the qualitative behaviour obtained already in the $\kappa$ parameter expansion at larger masses [12]. This has, of course, no direct implication for light fermions effects.

One of the authors (IOS) wants to thank P. Hasenfratz, C. Lang and V. Linke for discussions and remarks.

\section{References}

[1] G. 't Hooft, Recent developments in gauge theory (Cargèse, 1979), ed. G. 't Hooft (Plenum, New York, 1980).

[2] K. Osterwalder and E. Seiler, Ann. Phys. (NY) 110 (1978) 440.

[3] E. Fradkin and D. Shenker, Phys. Rev. D19 (1979) 3682.

[4] A. Guth, Phys. Rev. D23 (1981) 347.

[5] A. Albrecht and P. Steinhardt, Phys. Rev. Lett. 48 (1982) 1220.

[6] F. Karsch, E. Seiler and I.O. Stamatescu, Phys. Lett. $131 \mathrm{~B}(1983) 138$.

[7] T. Munehisa and Y. Munehisa, Nucl. Phys. B215 [FS7] (1983) 508.

[8] H. Kuhnelt, C.B. Lang and G. Vones, Nucl. Phys. B230 [FS10] (1984) 31.

[9] V.P. Gerdt, A.S. Ilchev, V.K. Mitryushkin, I.K. Sobolev and A.M. Zadorozhny, preprint Dubna E2-84-313 (1984).

[10] C. Hege, V. Linke, E. Seiler and I.O. Stamatescu, work in progress.

[11] G. Mack, Commun. Math. Phys. 65 (1979) 91.

[12] P. Hasenfratz, F. Karsch and I.O. Stamatescu, Phys. Lett. 133B (1983) 221.

[13] J. Polonyi, H.W. Wyld, J.B. Kogut, J. Shigemitsu and D.K. Sinclair, Phys. Rev. Lett. 53 (1984) 644.

[14] T. Çelik, J. Engels and H. Satz, Phys. Lett. 133B (1984) 427.

[15] I.O. Stamatescu, preprint MPI, MPI-PAE-PTh 92/83 (1983). 